\title{
Methylphenidate Normalizes Resting-State Brain Dysfunction in Boys With Attention Deficit Hyperactivity Disorder
}

\author{
Li An ${ }^{1,2}$, Xiao-Hua Cao,,2, Qing-Jiu Cao, ${ }^{1,2}$, Li Sun ${ }^{1,2}$, Li Yang ${ }^{1,2}$, Qi-Hong Zou ${ }^{3}$, Rubia Katya ${ }^{4}$, \\ Yu-Feng Zang*,5,6 and Yu-Feng Wang*,1,2 \\ IInstitute of Mental Health, Peking University, Beijing, China; ${ }^{2}$ Key Laboratory of Mental Health, Ministry of Health, Peking University, Beijing, \\ China; ${ }^{3}$ MRI Research Center and Beijing Key Lab of Medical Physics and Engineering, Peking University, Beijing, China; ${ }^{4}$ Department of \\ Child Psychiatry, Institute of Psychiatry, King's College London, London, UK; ${ }^{5}$ Center for Cognition and Brain Disorders, Affiliated Hospital, \\ Hangzhou Normal University, Hangzhou, Zhejiang, China; ${ }^{6}$ State Key Laboratory of Cognitive Neuroscience and Learning, Beijing Normal \\ University, Beijing, China
}

We used resting-state functional magnetic resonance imaging (RS-fMRI) to investigate the acute effects of methylphenidate hydrochloride (MPH) on spontaneous brain activity in children with attention deficit hyperactivity disorder (ADHD). In all, 23 boys with ADHD were scanned twice, under either $10 \mathrm{mg}$ dose of $\mathrm{MPH}$ or placebo, in a randomized, cross-over, counterbalanced placebocontrolled design. 32 Matched healthy controls were scanned once for comparison. Seven of the 23 ADHD boys participated in a followup 8-week $\mathrm{MPH}$ treatment. A regional homogeneity (ReHo) method was applied to characterize the local synchronization of spontaneous brain activity. ADHD boys under placebo compared with controls showed decreased ReHo in bilateral dorsolateral prefrontal cortices and increased ReHo in bilateral sensorimotor and parieto-visual cortices. Relative to placebo, MPH upregulated ReHo in bilateral ventral prefrontal cortices and cerebellar vermis, and downregulated ReHo in right parietal and visual areas that overlapped with the abnormally enhanced activities. When under $\mathrm{MPH}$, ReHo differences between patients and controls were no longer observed. The preliminary prediction analysis revealed that the decreased ReHo in right parietal cortex after the acute MPH was positively correlated with the decreased symptom scores after the 8-week MPH treatment in the seven patients. We show that an acute dose of $\mathrm{MPH}$ normalized all fronto-parieto-cerebellar dysfunctions in boys with ADHD during the resting state. Preliminary findings furthermore suggest the potential of RS-fMRI as a prognostic imaging tool to identify response to MPH treatment.

Neuropsychopharmacology (2013) 38, 1287-1295; doi:I0.1038/npp.2013.27; published online 27 February 2013

Keywords: methylphenidate hydrochloride (MPH); attention deficit hyperactivity disorder (ADHD); resting state; functional magnetic resonance imaging ( $\mathrm{MMRI}$ ); regional homogeneity (ReHo); treatment response prediction.

\section{INTRODUCTION}

Attention deficit hyperactivity disorder (ADHD) is one of the most commonly diagnosed childhood neurobehavioral disorders, affecting $5-8 \%$ of school-age children (Biederman and Faraone, 2005). Methylphenidate hydrochloride (MPH) has been used as the first-line treatment of ADHD for $>50$ years. However, the neural basis for its therapeutic effect still remains unclear. Most evidence for baseline cerebral functional change elicited by MPH has come from single photon emission computerized tomogra-

\footnotetext{
*Correspondence: Professor YF Wang, Institute of Mental Health, Peking University, 5I Huayuan North Road, Haidian District, Beijing I00191, China, Tel: + 86010 82802907, Fax: + 8601062070258 , E-mail: wangy@bjmu.edu.cn

Or Professor YF Zang, Center for Cognition and Brain Disorders, Hangzhou Normal University, Room 26I, Building 7, The Affiliated Hospital of Hangzhou Normal University, No. 126, Wenzhou Road, Hangzhou, Zhejiang 3100I5, China, Tel: + 86 57I 88285650,

Fax: + 86 57। 8828565 I, E-mail: zangyfgmail.com

Received 25 September 2012; revised 24 December 2012; accepted 7 January 2013; accepted article preview online 22 January 2013
}

phy (SPECT) and positron emission tomography (PET) studies showing that MPH impacts most prominently on fronto-striatal-parieto-cerebellar circuits, which are also the underlying neural basis of ADHD pathophysiology (Dickstein et al, 2006). The use of SPECT and PET techniques in children and adolescent is, however, constrained by ethical considerations associated with their ionizing radiation. Due to the non-invasiveness and a fairly high temporal and spatial resolution, resting-state functional magnetic resonance imaging (RS-fMRI) techniques, including blood oxygen level dependent (BOLD) and arterial spin labeling (ASL), have been largely used in the last decade to explore functional abnormalities of ADHD adults and children. To date, however, there is only one published RS-fMRI study in adult ADHD using ASL, that found that over 1-month stimulant treatment normalized hyperperfusion in nine ADHD adults in the left caudate, while it ameliorated hyperperfusion in the left ventral prefrontal cortex (PFC) (O'Gorman et al, 2008), which is opposite to short- and long-term BOLD-fMRI effects of MPH of upregulating fronto-striatal activation in 
children with ADHD (Epstein et al, 2007; Konrad et al, 2007; Rubia et al, 2009b, 2011a, 2011b; Vaidya et al, 1998). However, there may be neurofunctional differences between ADHD adults and children (Epstein et al, 2007) as well as between BOLD and ASL measures (Ogawa et al, 1993), and it is hence paramount to explore the MPH effects on the baseline brain function of ADHD children using RS-fMRI techniques.

Regional homogeneity (ReHo) is a newly-developed approach for the measurement of local synchronization of BOLD RS-fMRI signals. It calculates the temporal similarity of time series of a given voxel to those of its nearest neighboring voxels with the hypothesis that the time series in a functional cluster should be highly synchronous (Zang et al, 2004). ReHo methods have been used to explore functional abnormalities of many brain disorders. For example, regions exhibiting increased $\mathrm{ReHo}$ in patients with epilepsy were in line with a hypothesis of increased local synchronization of epileptic discharges (Mankinen et al, 2011; Zhong et al, 2011). Adopting ReHo approach, our research group found that 29 ADHD boys showed decreased ReHo in underlying frontal-striatal-cerebellar circuits relative to healthy controls, indicating that the ReHo may be a potentially useful method in exploring the underlying pathophysiology of ADHD (Cao et al, 2006).

In this study, we therefore used ReHo combined with a whole-brain voxel-wise analysis to test the acute effects of a single dose of MPH on the spontaneous brain activity of ADHD children, in a randomized, cross-over, placebocontrolled RS-fMRI study. In addition, to examine the potential amelioration/normalization effects of MPH on abnormal brain function in ADHD patients relative to controls, we compared ReHo of the pediatric ADHD group under both the placebo and the MPH conditions with that of a matched control group. Lastly, in order to explore in a preliminary manner whether acute effects of MPH on ADHD brain function can predict treatment response, correlation analyses were performed between the acute change of spontaneous brain activity induced by MPH and the clinical efficacy after a later 8-week MPH treatment in a small subgroup of patients who received the 8-week MPH treatment after the scan.

\section{MATERIALS AND METHODS}

\section{Participants}

Participants included 23 right-handed boys diagnosed with ADHD (age range: 9-15 years, mean $=12.5 \pm 1.8$ ) and 32 healthy male controls matched in age and handedness (age range: $8-14$ years, mean $=11.8 \pm 1.8$ ). Exclusion criteria for the ADHD group were comorbidity with other current psychiatric disorders, except for conduct disorder (CD) or oppositional defiant disorder (ODD), as well as neurological abnormalities, drug or substance abuse, and full-scale IQ $<80$ measured by the Wechsler Intelligence Scale for Chinese Children-Revised (WISCC-R) (Gong and Cai, 1993). ADHD diagnosis was first made by a child psychiatrist, and then validated via a semistructured diagnostic interview, the Schedule for Affective Disorders and Schizophrenia for School-Age Children-Present and Lifetime Version (K-SADS-PL) (Kaufman et al, 1997), which is based on DSM-IV. The control boys were also interviewed with K-SADS-PL to ensure that they were excluded from the diagnosis of ADHD and other Axis I psychiatric disorders. For all subjects, baseline scores of the ADHD Rating ScaleIV (ADHD RS-IV) (DuPaul et al, 1998) and the Conners Parent Rating Scale (CPRS) (Goyette et al, 1978) were reported by the parents (see details in the Supplementary material).

Children with ADHD were scanned twice, in a singleblind, randomized, cross-over, placebo-controlled, and counterbalanced way. The two scans were 2-7 days apart (longer than five half lives of MPH), and each scan was taken $1 \mathrm{~h}$ after either $10 \mathrm{mg}$ dose of immediate-release MPH administration or placebo (Vitamin B6, $10 \mathrm{mg}$ ). Patients were stimulant-medication free for at least 1 month before the fMRI scan. The control boys were scanned once without MPH or placebo. Written informed consent was obtained from each parent and each child agreed to participate. The local ethical committee approved this study.

The final statistical analyses were based on the data of 22 patients and 30 controls (see Data analysis). Among the 22 patients, five were comorbid with ODD; 15 patients were psychostimulant-naive and the other seven had a history of stimulant administration (three of shorter than 2 weeks, 3 of 3-6 months, and 1 of 17 months); 10 patients met the criteria for the predominantly inattentive subtype, and the other 12 met the criteria for the combined (inattentive/ hyperactive-impulsive) subtype.

After the second scan, seven of the 22 ADHD boys participated in a follow-up open-label 8-week MPH treatment. The MPH dose was escalated every week until optimal response was achieved or adverse effects could not be tolerated. ADHD RS-IV was rated for each patient every week by a senior child psychiatrist, based on both parent and teacher reports. The mean decrease in total score from baseline to the eighth week on the ADHD RS-IV for the seven patients was $16.86 \pm 11.13(P=0.007, t=4.01)$. The mean score decrease rate was $49.40 \pm 23.51 \%$ (15.38-83.78\%).

\section{Data Acquisition}

Images were acquired on a Siemens Trio 3-Tesla scanner (Siemens, Erlangen, Germany) at the State Key Laboratory of Cognitive Neuroscience and Learning, Beijing Normal University. The RS-fMRI images were acquired using an echo-planar imaging (EPI) sequence with 33 axial slices, repetition time $(\mathrm{TR})=2000 \mathrm{~ms}$, echo time $(\mathrm{TE})=30 \mathrm{~ms}$, flip angle $=90^{\circ}$, slice thickness $/$ skip $=3.0 / 0.6 \mathrm{~mm}$, in-plane resolution $=64 \times 64,240$ volumes. High-resolution T1weighted structural images covering the whole brain were then acquired (see details in the Supplementary material). Other subsequent scanning sessions were not used in the current study.

\section{Data Analysis}

Image preprocessing, including slice timing, head motion correction, within-subject registration of RS-fMRI data and T1 images, and spatial normalization (voxel size $3 \times 3 \times 3$ $\mathrm{mm}^{3}$ ), was performed using SPM8 (http://www.fil.ion. ucl.ac.uk/spm). Participants (one ADHD and two controls) with head motion $>3 \mathrm{~mm}$ of translation or $>3$ degree of 
rotation in any direction were excluded from further analysis. Resting-State fMRI Data Analysis Toolkit (REST) (Song et al, 2011) was then used for the following steps. The linear trend of the fMRI data was removed, and band-pass filtering $(0.01-0.08 \mathrm{~Hz})$ was conducted to decrease the impact of high-frequency physiological noise and the very low-frequency drift.

Individual ReHo map was generated by calculating the Kendall coefficient of concordance (KCC) of the time series of a given voxel with those of its neighbors (26 voxels) in a voxel-wise way (Kendall and Gibbons, 1990; Zang et al, 2004). Afterwards a whole-brain mask (70831 voxels; made from the MNI template) was adopted to remove the nonbrain tissues. For standardization purposes, the individual ReHo maps were divided by their own global mean KCC within the whole-brain mask. Then spatial smoothing was performed on the standardized individual ReHo maps with a Gaussian kernel of $6 \mathrm{~mm}$ full-width at half-maximum.

\section{Statistical Analyses}

Case-control differences in age and full-scale IQ were calculated in SPSS13.0 (http://www.spss.com). For ReHo, two two-sample $t$-tests between the ADHD group under the placebo condition/ the MPH condition and the control group were conducted in a whole-brain voxel-wise way using SPM8. Then a voxel-wise paired $t$-test was performed to examine the differences between the placebo condition and the MPH condition in the ADHD group. Voxels with $P<0.01$ and cluster size $>1080 \mathrm{~mm}^{3}$, which resulted in a corrected threshold of $P<0.05$ determined by AlphaSim in AFNI ( $\mathrm{rmm}=5$; http://afni.nih.gov/afni/ docpdf/AlphaSim.pdf), were regarded to show a significant difference between the two conditions or the two groups.

Among the seven patients who received the later 8-week MPH treatment, two correlation analyses were conducted in REST within the brain regions showing significant $\mathrm{MPH}$ effect on ADHD in a voxel-wise way between the eighthweek score of ADHD RS-IV and the ReHo under placebo condition/ the acute ReHo change (ie, placebo condition minus MPH condition), when covarying for the baseline score of ADHD RS-IV. The resultant correlation map was set at a combined threshold of $P<0.05$ and a minimum cluster size of $540 \mathrm{~mm}^{3}$ (corrected by AlphaSim).

\section{RESULTS}

\section{Demographic Characteristics}

There were no significant group differences for age ( $t=1.12, P=0.27)$. However, the full-scale IQ of the ADHD group was lower than that of the control group (ADHD: $110.5 \pm 12.5$, Control: $121.0 \pm 12.7 \quad(n=29,1$ missing $)$; $t=-2.94, P=0.005)$.

\section{RS-fMRI Data}

Motion. Univariate analyses of variance (ANOVAs) revealed no significant group differences between controls and ADHD children under either condition in total maximum or total mean translation or rotation in the $x$, $y$, and $z$ dimensions (Fs $(2,71)<2.24$, Ps $>0.11$ ).
Comparisons between controls and ADHD boys under either placebo or MPH condition. Under placebo, ADHD children relative to controls showed decreased ReHo in bilateral superior frontal gyrus, as well as increased ReHo in bilateral sensorimotor cortex, right premotor area, left cuneus, right calcarine, and superior occipital gyri (see Table 1 and Figure 1). While under $\mathrm{MPH}$, no significant ReHo differences were found between the two groups.

$\mathrm{ReHo}$ in right calcarine gyrus under placebo was marginally positively correlated with the hyperactivity/ impulsivity score of ADHD RS-IV $(r=0.40, P=0.06)$ in the ADHD group. ReHo in the right premotor area was positively correlated with the impulsive-hyperactive factor $(r=0.53, P=0.01)$ and the hyperactive index factor ( $r=0.40, P=0.07$, marginally significant) of the CPRS in ADHD (see details in the Supplementary materials).

Low IQ is associated with ADHD (Dennis et al, 2009). It was proposed that, when the covariate is intrinsic to the condition and differs between groups, it is inappropriate to correct group effects for differences in the covariate because the group effect would be altered in potentially problematic ways, leading to artifactual results (Dennis et al, 2009; Miller and Chapman, 2001). For this concern, we first did not take IQ as covariate when comparing between the two groups. However, to assess potential effects of IQ on the results, we performed post hoc analyses to examine the linear relationship between IQ and mean ReHo within areas that differed between groups. No significant correlations were found in either group between IQ and any area that differed between groups, except for left cuneus $(r=-0.46$, $P=0.01)$ and right calcarine gyrus $(r=-0.42, P=0.03)$ both of which were significantly associated with IQ in controls. We then re-performed two-sample $t$-tests to investigate to how much extent the IQ would affect the between-group differences aforementioned. The results showed that there were still no significant ReHo differences found between the boys with ADHD under MPH and controls. In placebo condition for ADHD, two clusters with decreased ReHo in superior frontal gyrus disappeared.

Table I Clusters of Significant ReHo Differences Between ADHD Group Under Placebo Condition and Control Group

\begin{tabular}{|c|c|c|c|c|c|c|c|}
\hline \multirow[t]{2}{*}{ Areas } & \multirow[t]{2}{*}{ BA } & \multirow{2}{*}{ L/R } & \multirow{2}{*}{$\begin{array}{l}\text { Volume } \\
\left(\mathrm{mm}^{3}\right)\end{array}$} & \multicolumn{3}{|c|}{ MNI } & \multirow[t]{2}{*}{ t-value } \\
\hline & & & & $\mathbf{x}$ & y & $\mathbf{z}$ & \\
\hline \multicolumn{8}{|l|}{$A D H D$ group $<$ control group } \\
\hline Superior frontal gyrus & 10 & $\mathrm{~L}$ & $170 \mid$ & -18 & 54 & 15 & -4.00 \\
\hline Superior frontal gyrus & $32 / 8 / 9$ & $\mathrm{R}$ & $170 \mid$ & 18 & 24 & 45 & -3.53 \\
\hline \multicolumn{8}{|c|}{$A D H D$ group $>$ control group } \\
\hline Cuneus & $19 / 18$ & L & 2889 & -12 & $-8 \mid$ & 24 & 5.07 \\
\hline \multirow{2}{*}{ Sensorimotor cortex ${ }^{a}$} & 6 & L & \multirow{2}{*}{9909} & -3 & -18 & 57 & 4.26 \\
\hline & 3 & $\mathrm{R}$ & & 21 & -39 & 66 & 3.93 \\
\hline Premotor area & 6 & $\mathrm{R}$ & $116 \mid$ & 18 & -9 & 66 & 3.78 \\
\hline Calcarine gyrus & 18 & $\mathrm{R}$ & 1242 & 18 & -63 & 18 & 3.60 \\
\hline Postcentral gyrus & 3 & $\mathrm{~L}$ & 1188 & -45 & -30 & 66 & 3.59 \\
\hline Superior occipital gyrus & $17 / 18$ & $\mathrm{R}$ & 2187 & 15 & -102 & 6 & 3.16 \\
\hline
\end{tabular}

Abbreviations: BA, Brodman area; $M N I, M N I$ coordinates; $R$, right; $L$, left.

${ }^{a}$ Areas in bilateral sensorimotor cortex merged into one cluster. 
However, when a slightly more lenient threshold of $P<0.01$ and cluster size $>810 \mathrm{~mm}^{3}$ (uncorrected) was applied, the pattern of between-group difference was almost the same as that of without IQ covariate (see details in the Supplementary materials; corrected for multiple comparison: Supplementary Table S1 and Supplementary Figure S1; uncorrected: Supplementary Table S2 and Supplementary Figure S2).

The six motion parameters were further treated as covariates to investigate their effects on between-group ReHo difference. The results were quite similar as those without taking head motion as covariates. The case was the same for the following within-patient comparison analysis (see details in the Supplementary materials; ADHD on placebo vs controls: Supplementary Table S3 and Supplementary Figure S3; ADHD on MPH vs controls: Supplementary Figure S4; MPH vs placebo in ADHD: Supplementary Table S4 and Supplementary Figure S5).

Within-patient comparison between the placebo and the $\mathrm{MPH}$ condition. Compared with placebo, MPH decreased $\mathrm{ReHo}$ in the right lingual gyrus and right postcentral gyrus (extending to the superior and inferior parietal lobule), and increased ReHo in the left inferior frontal cortex (IFC), right orbital frontal cortex (OFC), and the cerebellar vermis (Table 2; Figure 2).

There was a significant negative correlation between the impulsive-hyperactive factor of the CPRS and ReHo under placebo in the cerebellar vermis $(r=-0.45, P=0.04)$ and a marginal positive correlation between the impulsive-hyperactive factor of the CPRS and ReHo in right parietal cortex
( $r=0.40, P=0.07)$ as well as right $\mathrm{OFC}(r=0.41, P=0.06)$ in ADHD.

Conjunction analyses. The conjunction analysis revealed a common cluster in right postcentral and superior parietal lobe (BA 3/5, 77 voxels), which was significantly downregulated with $\mathrm{MPH}$ within the ADHD group and, in addition, was hyperactive in patients relative to controls and then normalized with MPH (see details in the Supplementary materials; Supplementary Figure S6).

Correlation analyses. Among the seven patients who received the later 8 -week $\mathrm{MPH}$ treatment, the decrease in

Table 2 Clusters of Significant ReHo Differences Between MPH Condition and Placebo Condition in the ADHD Group

\begin{tabular}{|c|c|c|c|c|c|c|c|}
\hline \multirow[t]{2}{*}{ Areas } & \multirow[t]{2}{*}{ BA } & \multirow[t]{2}{*}{ L/R } & \multirow{2}{*}{$\begin{array}{l}\text { Volume } \\
\left(\mathrm{mm}^{3}\right)\end{array}$} & \multicolumn{3}{|c|}{ MNI } & \multirow[t]{2}{*}{$t$-value } \\
\hline & & & & $x$ & $y$ & $\mathbf{z}$ & \\
\hline \multicolumn{8}{|c|}{ MPH condition < placebo condition } \\
\hline Lingual gyrus & $17 / 18 / 19$ & $\mathrm{R}$ & 2376 & 6 & -75 & -12 & -5.14 \\
\hline $\begin{array}{l}\text { Right postcentral gyrus } \\
\text { (extending to the superior } \\
\text { and inferior parietal lobule) }\end{array}$ & 3/40/7 & $\mathrm{R}$ & 5913 & 33 & -33 & 54 & -4.37 \\
\hline \multicolumn{8}{|l|}{ MPH condition > placebo condition } \\
\hline Inferior frontal cortex & $45 / 47$ & $\mathrm{~L}$ & 1080 & -36 & 39 & 6 & 5.72 \\
\hline Orbitofrontal cortex & | | & $\mathrm{R}$ & 1188 & 21 & 39 & -12 & 4.76 \\
\hline Cerebellar vermis & & & 2079 & 0 & -54 & -30 & 4.61 \\
\hline
\end{tabular}

Abbreviations: BA, Brodman area; MNI, MNI coordinates; $\mathrm{MPH}$, methylphenidate; $R$, right; $L$, left.

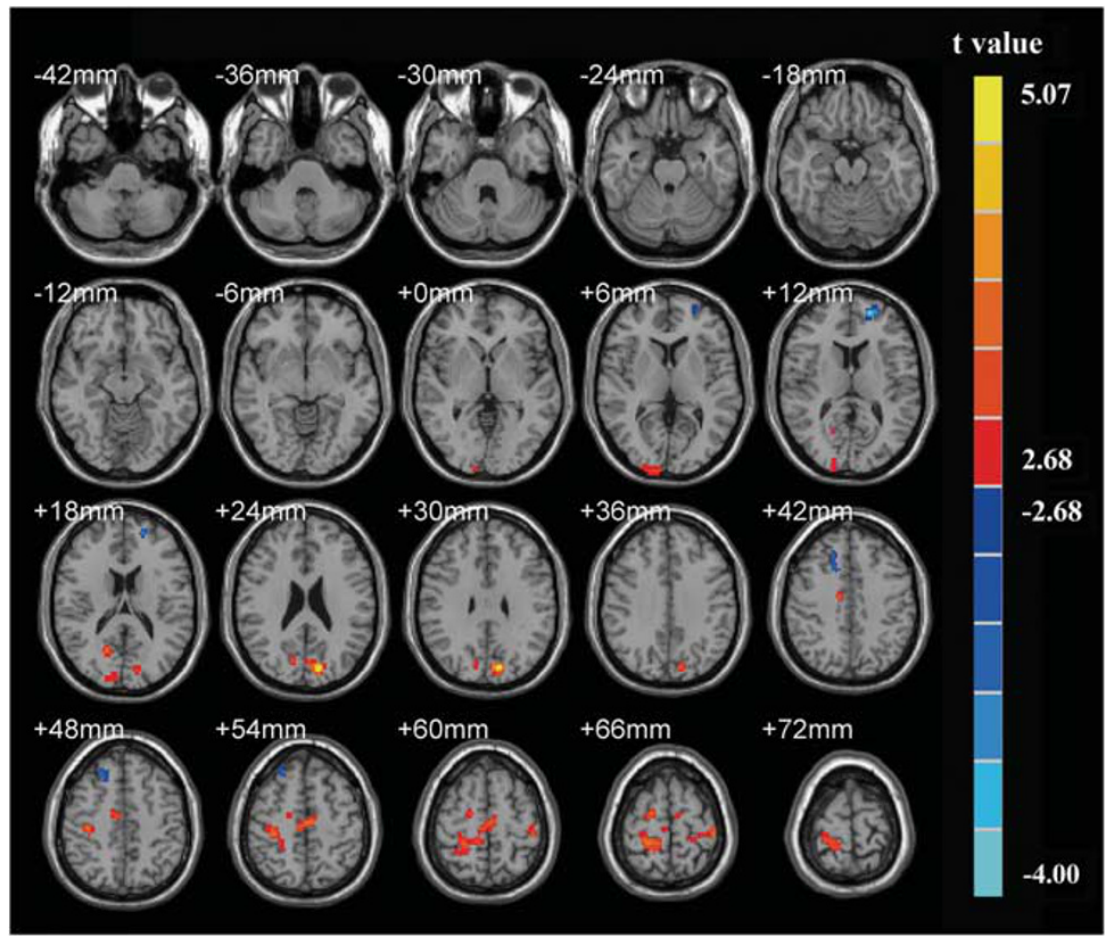

Figure I Clusters showing significant ReHo differences between ADHD group under placebo condition and control group. The cold colors indicate lower ReHo in ADHD group under placebo than control group, while the warm colors mean vice versa; all clusters $P<0.05$ (corrected). Left in the figure indicates the right side of the brain. 


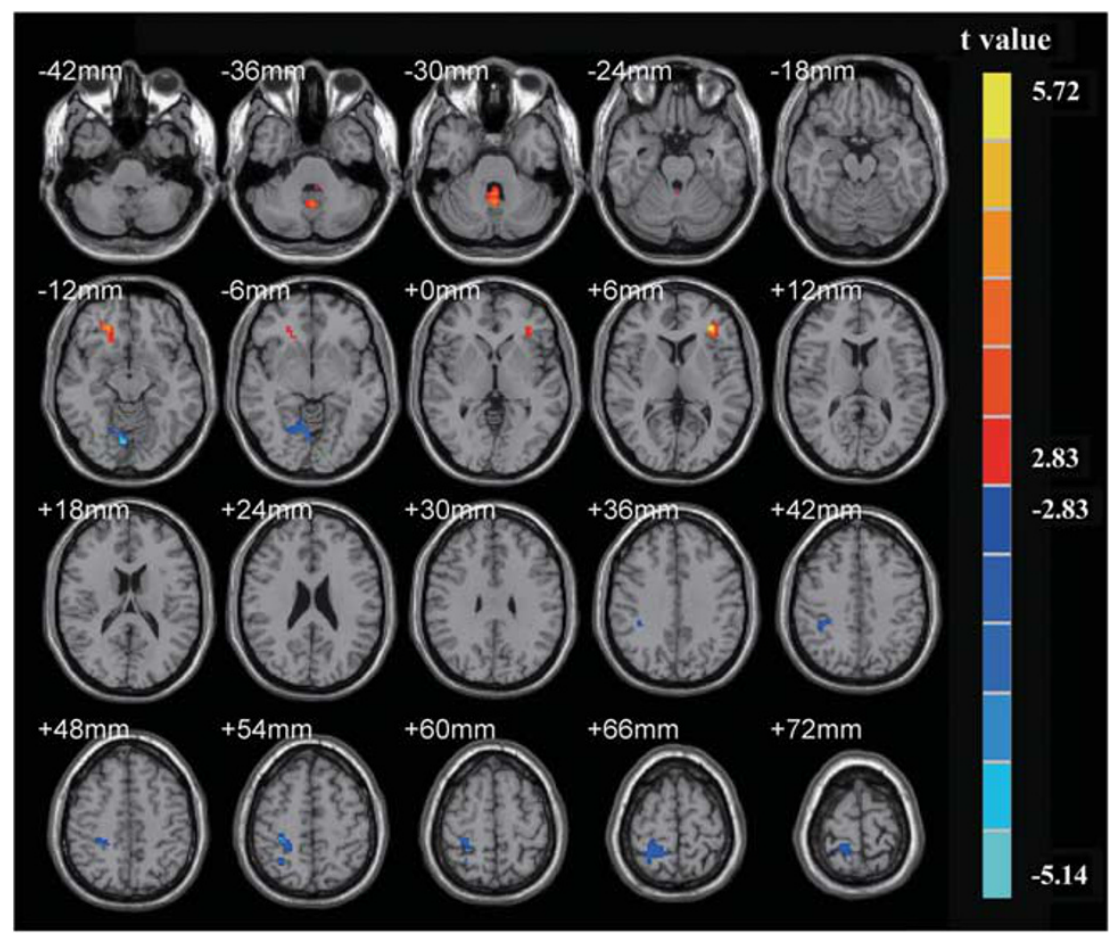

Figure 2 Clusters showing significant ReHo differences between MPH condition and placebo condition in the ADHD group. The cold colors indicate lower ReHo in MPH condition than placebo condition, while the warm colors mean vice versa; all clusters $P<0.05$ (corrected). Left in the figure indicates the right side of the brain.

ReHo by one single dose of MPH in the right postcentral gyrus and superior parietal lobe (BA 5/3/7, 37 voxels) was negatively correlated with the 8 th week scores of the ADHD RS-IV, covaried for the baseline scores $(r=-0.90$, $P=0.01$ ) (Figure 3).

There was no significant correlation between the ReHo under placebo in any brain region and the 8th week scores of ADHD RS-IV in the seven patients, covaried for the baseline scores.

\section{DISCUSSION}

In the current RS-fMRI study, ADHD children under placebo compared with controls showed decreased activity in bilateral frontal pole and dorsal lateral PFC (DLPFC), and increased activity in bilateral sensorimotor and parieto-visual cortices. Among patients, MPH relative to placebo significantly upregulated activity in left IFC, right OFC, and cerebellar vermis, and downregulated activity in right parietal and visual cortex that overlapped with the abnormally enhanced parieto-visual activity areas. When under $\mathrm{MPH}$, activity differences between patients and controls were no longer observed. Furthermore, the exploratory predictive analysis in a subgroup of ADHD patients showed that the decreased activity in a cluster of right parietal cortex, which was almost in the same location as the parietal cluster that was enhanced in ADHD under placebo and reduced by MPH in the whole group, by a single-dose MPH administration was positively correlated with the decreased symptom scores after the 8-week MPH treatment.

The neurophysiological basis of ReHo should be discussed first. Although ReHo has been used in many studies

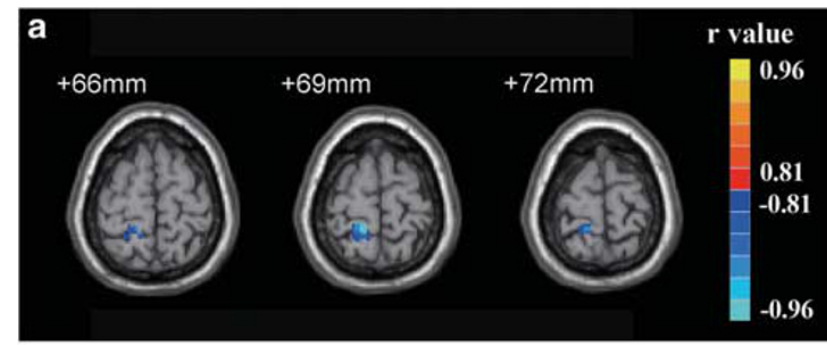

b

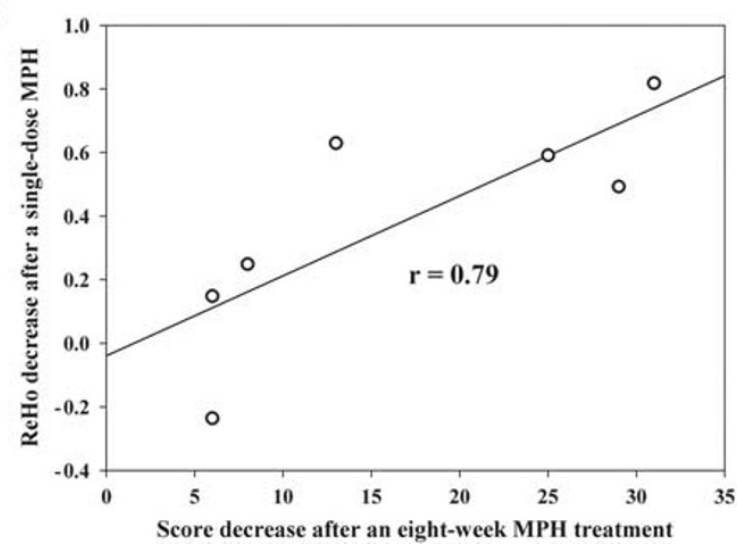

Figure 3 The ReHo decrease after the acute MPH administration in right postcentral gyrus and superior parietal lobe was negatively correlated with the eighth-week score of ADHD RS-IV in the seven patients, covarying for the baseline score. (a) Voxel-wise correlation map: the cluster is located in BA 5/3/7 with 37 voxels ( $M N I$ coordinates of the peak voxel: $12,-42,69)$. Left in the figure indicates the right side of the brain. (b) The scatter plot showed the positive correlation between the decreased mean ReHo with single-dose MPH extracted from the above cluster and the decreased score after 8-week MPH treatment: $r=0.79, P=0.04$. 
on brain disorders, its correlation with neuronal activity has only some indirect evidences. In the study in which ReHo was developed, it was found that finger tapping tasks increased the ReHo in the primary sensorimotor cortex but reduced the ReHo in the default mode network (Zang et al, 2004), a similar pattern of increased and decreased activity as found in task fMRI studies and PET studies (Raichle et al, 2001). Epilepsy is a representative model of abnormal spontaneous neuronal activity. A RS-fMRI study on patients with medial temporal lobe epilepsy found increased ReHo in the medial temporal lobe (Mankinen et al, 2011). Another RS-fMRI study showed increased ReHo in the thalamus in patients with generalized tonic-clonic seizures (Zhong et al, 2011). These studies suggested that increased spontaneous neuronal activity may underlie the increased ReHo or local synchronization of spontaneous low-frequency BOLD activity. Studies of simultanenous recording of BOLD and neuronal activity have shown that the local field potential (LFP) has close correlation with BOLD signal (Logothetis et al, 2001; Rauch et al, 2008). An electrophysiological study revealed high local synchronization between the LFPs of multiple cortical electrodes with a physical distance ranged from 2.6 to $10.6 \mathrm{~mm}$ and such local synchronization could be modulated by stimulation (Leopold et al, 2003). Based on all these above indirect evidences, we believe that ReHo is likely reflective of coordination of combined regional neuronal activity subserving similar goals or representations. In future studies, to measure the local synchronization of both LFP and BOLD signals by using simultaneous recording will help understand the electrophysiological mechanism of ReHo.

Consistent resting-state (Konrad and Eickhoff, 2010) and cognitive (Durston et al, 2011) neuroimaging evidences have shown hypofrontality in ADHD patients. And single doses of MPH have been associated with increased regional cerebral blood flow (rCBF) (Kim et al, 2001; Lee et al, 2005) or task activation (Epstein et al, 2007; Konrad et al, 2007; Rubia et al, 2009b, 2011a, 2011b; Vaidya et al, 1998) in PFC, in accordance with our RS-fMRI results of upregulation of bilateral dorsal-to-ventral regions of PFC. The DLPFC/IFC provides 'top-down' cognitive control and attention, inhibiting the processing of irrelevant stimuli, sustaining attention over time, and dividing, selecting and coordinating attention (Arnsten and Rubia, 2012). This frontal lobe mediated attention processes are opposite to the 'bottom-up' visual-spatial attention processes mediated by posterior parietal association cortices. The parietal association cortices process where visual stimuli are and whether the stimuli are moving, which originates from striate and extrastriate visual cortices (Culham and Kanwisher, 2001). The DLPFC/IFC and parietal cortices are intricately interconnected to provide unified and tightly regulated attention functions (Arnsten, 2009a; Arnsten and Rubia, 2012). Besides attention control, the IFC has also been strongly associated with inhibitory control, together with its connections to striatal and subthalamic nuclei (Chambers et al, 2009). Therefore, the baseline hyperactivity of the sensory and motor-related cortices in ADHD patients may be due to the inability to suppress responsiveness to irrelevant sensory stimuli in the environment and may be associated with hyperactivity (Lee et al, 2005), possibly related to reduced top-down control from DLPFC and IFC.
Our findings are in line with consistent evidence of hyperactive sensorimotor cortices and visual areas in ADHD during the resting state (Cao et al, 2006; Lee et al, 2005; O'Gorman et al, 2008; Schweitzer et al, 2003; Tian et al, 2008; Zang et al, 2007).

The suppressing effects of MPH on the BOLD signal of the sensorimotor and visual cortices in ADHD in the current results extend previous SPECT and PET studies that examined rCBF (Lee et al, 2005; Lou et al, 1984, 1989; O'Gorman et al, 2008; Schweitzer et al, 2003). It is possible that by upregulating the abnormally hypoactive DLPFC and IFC, as well as downregulating the abnormal hyperactivity of the sensory and motor-related cortices, MPH may assist ADHD patients in filtering unnecessary sensory stimuli and inhibiting relevant motor responsiveness, enabling them to engage active attention and top-down self-control and then organize their behavior (Schweitzer et al, 2003). This would be supported by the correlation between higher baseline activity in extensive parietal cortex, premotor and calcarine gyri in ADHD children and more severe hyperactivityimpulsivity symptoms.

The OFC is associated with reward processing and flexible regulation of emotional response to reward and punishment (Dias et al, 1996). It is reported to exhibit decreased nodal efficiency in small-world network typology (Wang et al, 2009) and reduced gray matter volume or myelination (Makris et al, 2008) in patients with ADHD. However, the OFC cluster in our study is partly located in white matter, which calls for treating the results from this region with caution.

MPH also upregulated activity in the cerebellar vermis in ADHD children in the current study, which extends previous findings of $\mathrm{MPH}$-related $\mathrm{rCBF}$ increase in the vermis of ADHD using relaxometry or PET (Anderson et al, 2002; Schweitzer et al, 2003). The PFC and parietal cortices project to the cerebellum by way of the pontine nuclei. And reduced IFC and cerebellar activations, in some studies along with reduced inter-regional functional connectivity were found in ADHD during vigilance (Rubia et al, 2009b), working memory (Sheridan et al, 2010), response inhibition (Rubia et al, 2011b), and time discrimination (Rubia et al, 2009a) and could be upregulated and normalized after one single dose of $\mathrm{MPH}$ during vigilance (Rubia et al, 2009b), response inhibition (Rubia et al, 2011b), and time discrimination (Rubia et al, 2009a). Along with the correlation finding between the lower baseline activity in cerebellar vermis in ADHD children and the more severe hyperactivity-impulsivity symptoms in our study, it is possible that MPH may promote complex cognitive functions such as attention, timing, and working memory as well as behavior control in ADHD by elevating both the regional activity and the functional connectivity within this fronto-parieto-cerebellar circuit.

The above-mentioned notion is further supported by the close correlation between the acute functional change in the right parietal cortex (including primary sensory and association cortices) by a single-dose MPH and the clinical efficacy of an 8-week MPH treatment found in the current study. This preliminary correlation finding, to our knowledge, shows for the first time, that there is a direct relationship between the acute change in local brain activity by a single dose of $\mathrm{MPH}$ and the clinical efficacy of an 
8-week MPH treatment. In 1995, Buitelaar et al (1995) had already shown that a positive response to a single dose of $\mathrm{MPH}$ is one of the two important contributors to $\mathrm{MPH}$ response prediction in ADHD children. The current study shows that those ADHD children who show a larger downregulated function by a single-dose $\mathrm{MPH}$ in right parietal cortex are more likely to respond to a later 8-week $\mathrm{MPH}$ treatment. This result is also consistent with the findings of a previous SPECT study (Cho et al, 2007), in which the ADHD children who did not respond to an 8-week MPH treatment exhibited lower baseline rCBF in the right superior parietal cortex relative to responders, highlighting the potential important role of the right parietal cortex in the therapeutic mechanism of MPH. We did, however, not find any correlation between the ReHo under placebo and the decreased symptom score after 8-week $\mathrm{MPH}$ treatment, which would have been more clinically useful. Nevertheless, the current preliminary correlation findings, if replicated in larger samples, may indicate the potential of RS-fMRI as a tool for identifying response to psychostimulant treatment based on brain modulation in an acute dose. Being able to predict whether an individual child will respond to chronic stimulant medication by one or two short scans of $8 \mathrm{~min}$ at baseline or/and under one single acute MPH dose would be highly useful to refine treatment decision, avoid delays in symptomatic improvement, and further treatment compliance. We are, however, aware of the small sample sizes and findings hence have to be considered preliminary.

Stimulants such as methylphenidate block reuptake of norepinephrine (NE) and dopamine (DA) into transporters, increasing the activity of these neurotransmitter systems. While high-dose stimulants mainly increase subcortical DA release (Kuczenski and Segal, 1997; Volkow et al, 2002), oral low-dose stimulants preferentially produce high-levels of $\mathrm{NE}$ increase, and significant levels of DA increase, in the PFC; however, they have less effect on DA release in subcortical areas (Berridge et al, 2006; Kuczenski and Segal, $2002,2005)$. This may partly explain why a relative low dose $(0.14-0.40 \mathrm{mg} / \mathrm{kg})$ of MPH did not elicit a significant functional change in striatal areas in ADHD children in our study. The function of the dorsal PFC is especially dependent on the levels of DA and NE in an 'inverted U' dosedependent way (Arnsten and Rubia, 2012). By stimulating postsynaptic alpha-2A adrenoceptors on PFC pyramidal cell spines, NE strengthens appropriate PFC network connections (increasing 'signals'); whereas by stimulating D1 receptor on a separate set of spines, DA shunts inappropriate network connections (decreasing 'noise') (Arnsten, 2009b). Although the amount of studies are still small, emerging data indicate that $\mathrm{NE}$ also has beneficial influence on IFC and OFC function; while DA appears to have a complex effect on the function of OFC (Arnsten and Rubia, 2012; Robbins and Arnsten, 2009).

IQ was lower in ADHD children in our study, which is typical for this population. We performed two-sample $t$-tests both with and without covarying IQ. The results showed that covarying IQ reduced the between-group difference to a small extent. It has been suggested that IQ-covariation would have been statistically inappropriate when the covariate is intrinsic to the condition and differs between groups (Dennis et al, 2009; Miller and Chapman,
2001). We would suggest, for ADHD studies, it may be more helpful to show results both with and without covarying IQ than doing only one of them.

The findings and interpretations of this study should be considered in light of its limitations. First, only seven patients were included to predict the 8-week MPH effects by single-dose $\mathrm{MPH}$-induced ReHo change, limiting generalization of the findings. Second, there were seven ADHD children in our patient sample who had a history of stimulant treatment and four of the seven patients with a stimulant history longer than 3 months. This, could have been a confounding effect on our results. Third, for reasons of ethics and feasibility, the control boys were not administered with either MPH or placebo and were scanned only once. Therefore, the potential confounding effects from placebo or scanning order cannot be excluded. Finally, ReHo algorithm measures the local synchronization. It has inherent spatial smoothness and therefore biases the estimation of spatial smoothness for multiple comparison correction (Tian et al, 2012). How to estimate the spatial smoothness is still an open issue. Therefore, we did not estimate the smoothness and used a simple smoothing kernel $(6 \mathrm{~mm})$ for AlphaSim multiple comparison correction. Future methodological studies are needed to estimate the intrinsic smoothing effect of ReHo.

To our knowledge, this is the first study using RS-fMRI technique to explore the effect of MPH on spontaneous brain activity of children with ADHD and to show that a single low dose of MPH normalized all the dysfunctions in fronto-parieto-cerebellar circuitry in ADHD relative to controls during the resting state. Furthermore, preliminary data showed that the downregulation of right parietal activity by a single dose of MPH significantly predicted the positive clinical response to an 8-week MPH treatment, indicating the possible important role of this area in the therapeutic mechanism of action of $\mathrm{MPH}$ and the possible potential of RS-fMRI as a prognostic imaging tool to identify response to pharmacological treatment.

\section{ACKNOWLEDGEMENTS}

The research was supported by the Commonweal Sciences Foundation, Ministry of Health, China (Grant number: 200802073), the National Foundation, Ministry of Science and Technology, China (Grant number: 2007BAI17B03), the National Natural Sciences Foundation, China (30970802, 81000593, 81020108022), and Open Research Fund of the State Key Laboratory of Cognitive Neuroscience and Learning, Beijing Normal University, China.

\section{DISCLOSURE}

Dr Li Yang received research grant from Janssen Science Council of China; she had been a speaker for Janssen and received travel support from Eli Lilly. Professor Katya Rubia has received funding from Lilly Pharmaceuticals for another project and speaker's honoraria from Lilly, Medice, and Novartis. Professor Yu-Feng Wang has served on an advisory board and received funding for research, lectures, and travel from Xi'an Janssen Pharmaceutical and the advisor of Eli Lilly and Company. 


\section{REFERENCES}

Anderson CM, Polcari A, Lowen SB, Renshaw PF, Teicher MH (2002). Effects of methylphenidate on functional magnetic resonance relaxometry of the cerebellar vermis in boys with ADHD. Am J Psychiatry 159: 1322-1328.

Arnsten AF (2009a). The emerging neurobiology of attention deficit hyperactivity disorder: the key role of the prefrontal association cortex. J Pediatr 154: 43.

Arnsten AF (2009b). Stress signalling pathways that impair prefrontal cortex structure and function. Nat Rev Neurosci 10: 410-422.

Arnsten AF, Rubia K (2012). Neurobiological circuits regulating attention, cognitive control, motivation, and emotion: disruptions in neurodevelopmental psychiatric disorders. J Am Acad Child Adolesc Psychiatry 51: 356-367.

Berridge CW, Devilbiss DM, Andrzejewski ME, Arnsten AF, Kelley AE, Schmeichel B et al (2006). Methylphenidate preferentially increases catecholamine neurotransmission within the prefrontal cortex at low doses that enhance cognitive function. Biol Psychiatry 60: 1111-1120.

Biederman J, Faraone SV (2005). Attention-deficit hyperactivity disorder. Lancet 366: 237-248.

Buitelaar JK, Van der Gaag RJ, Swaab-Barneveld H, Kuiper M (1995). Prediction of clinical response to methylphenidate in children with attention-deficit hyperactivity disorder. J Am Acad Child Adolesc Psychiatry 34: 1025-1032.

Cao Q, Zang Y, Sun L, Sui M, Long X, Zou Q et al (2006). Abnormal neural activity in children with attention deficit hyperactivity disorder: a resting-state functional magnetic resonance imaging study. Neuroreport 17: 1033-1036.

Chambers CD, Garavan H, Bellgrove MA (2009). Insights into the neural basis of response inhibition from cognitive and clinical neuroscience. Neurosci Biobehav Rev 33: 631-646.

Cho SC, Hwang JW, Kim BN, Lee HY, Kim HW, Lee JS et al (2007). The relationship between regional cerebral blood flow and response to methylphenidate in children with attention-deficit hyperactivity disorder: comparison between non-responders to methylphenidate and responders. J Psychiatr Res 41: 459-465.

Culham JC, Kanwisher NG (2001). Neuroimaging of cognitive functions in human parietal cortex. Curr Opin Neurobiol 11: 157-163.

Dennis M, Francis DJ, Cirino PT, Schachar R, Barnes MA, Fletcher JM (2009). Why IQ is not a covariate in cognitive studies of neurodevelopmental disorders. J Int Neuropsychol Soc 15: 331-343.

Dias R, Robbins TW, Roberts AC (1996). Dissociation in prefrontal cortex of affective and attentional shifts. Nature 380: 69-72.

Dickstein SG, Bannon K, Castellanos FX, Milham MP (2006). The neural correlates of attention deficit hyperactivity disorder: an ALE meta-analysis. J Child Psychol Psychiatry 47: 1051-1062.

DuPaul GJ, Power TJ, Anastopoulos AD, Reid R (1998). ADHD Rating Scale-IV: Checklists, Norms, and Clinical Interpretations. Guilford: New York.

Durston S, van Belle J, de Zeeuw P (2011). Differentiating frontostriatal and fronto-cerebellar circuits in attention-deficit/ hyperactivity disorder. Biol Psychiatry 69: 1178-1184.

Epstein JN, Casey BJ, Tonev ST, Davidson MC, Reiss AL, Garrett A et al (2007). ADHD- and medication-related brain activation effects in concordantly affected parent-child dyads with ADHD. J Child Psychol Psychiatry 48: 899-913.

Gong YX, Cai TS (1993). Manual of Chinese revised Wechsler Intelligence Scale for Children. Hunan Atlas Publishing House: Changsha.

Goyette CH, Conners CK, Ulrich RF (1978). Normative data on revised Conners Parent and Teacher Rating Scales. J Abnorm Child Psychol 6: 221-236.

Kaufman J, Birmaher B, Brent D, Rao U, Flynn C, Moreci P et al (1997). Schedule for affective disorders and schizophrenia for school-age children-present and lifetime version (K-SADS-PL): initial reliability and validity data. J Am Acad Child Adolesc Psychiatry 36: 980-988.

Kendall M, Gibbons J (1990). Rank Correlation Methods. Oxford University Press: Oxford.

Kim BN, Lee JS, Cho SC, Lee DS (2001). Methylphenidate increased regional cerebral blood flow in subjects with attention deficit/ hyperactivity disorder. Yonsei Med J 42: 19-29.

Konrad K, Eickhoff SB (2010). Is the ADHD brain wired differently? A review on structural and functional connectivity in attention deficit hyperactivity disorder. Hum Brain Mapp 31: 904-916.

Konrad K, Neufang S, Fink GR, Herpertz-Dahlmann B (2007). Long-term effects of methylphenidate on neural networks associated with executive attention in children with ADHD: results from a longitudinal functional MRI study. J Am Acad Child Adolesc Psychiatry 46: 1633-1641.

Kuczenski R, Segal DS (1997). Effects of methylphenidate on extracellular dopamine, serotonin, and norepinephrine: comparison with amphetamine. J Neurochem 68: 2032-2037.

Kuczenski R, Segal DS (2002). Exposure of adolescent rats to oral methylphenidate: preferential effects on extracellular norepinephrine and absence of sensitization and cross-sensitization to methamphetamine. J Neurosci 22: 7264-7271.

Kuczenski R, Segal DS (2005). Stimulant actions in rodents: implications for attention-deficit/hyperactivity disorder treatment and potential substance abuse. Biol Psychiatry 57: 1391-1396.

Lee JS, Kim BN, Kang E, Lee DS, Kim YK, Chung JK et al (2005). Regional cerebral blood flow in children with attention deficit hyperactivity disorder: comparison before and after methylphenidate treatment. Hum Brain Mapp 24: 157-164.

Leopold DA, Murayama Y, Logothetis NK (2003). Very slow activity fluctuations in monkey visual cortex: implications for functional brain imaging. Cereb Cortex 13: 422-433.

Logothetis NK, Pauls J, Augath M, Trinath T, Oeltermann A (2001). Neurophysiological investigation of the basis of the fMRI signal. Nature 412: 150-157.

Lou HC, Henriksen L, Bruhn P (1984). Focal cerebral hypoperfusion in children with dysphasia and/or attention deficit disorder. Arch Neurol 41: 825-829.

Lou HC, Henriksen L, Bruhn P, Borner H, Nielsen JB (1989). Striatal dysfunction in attention deficit and hyperkinetic disorder. Arch Neurol 46: 48-52.

Makris N, Buka SL, Biederman J, Papadimitriou GM, Hodge SM, Valera EM et al (2008). Attention and executive systems abnormalities in adults with childhood ADHD: A DT-MRI study of connections. Cereb Cortex 18: 1210-1220.

Mankinen K, Long XY, Paakki JJ, Harila M, Rytky S, Tervonen O et al (2011). Alterations in regional homogeneity of baseline brain activity in pediatric temporal lobe epilepsy. Brain Res 1373: 221-229.

Miller GA, Chapman JP (2001). Misunderstanding analysis of covariance. J Abnorm Psychol 110: 40-48.

Ogawa S, Menon RS, Tank DW, Kim SG, Merkle H, Ellermann JM et al (1993). Functional brain mapping by blood oxygenation level-dependent contrast magnetic resonance imaging. A comparison of signal characteristics with a biophysical model. Biophys J 64: 803-812.

O'Gorman RL, Mehta MA, Asherson P, Zelaya FO, Brookes KJ, Toone BK et al (2008). Increased cerebral perfusion in adult attention deficit hyperactivity disorder is normalised by stimulant treatment: a non-invasive MRI pilot study. Neuroimage 42: 36-41.

Raichle ME, MacLeod AM, Snyder AZ, Powers WJ, Gusnard DA, Shulman GL (2001). A default mode of brain function. Proc Natl Acad Sci USA 98: 676-682. 
Rauch A, Rainer G, Logothetis NK (2008). The effect of a serotonin-induced dissociation between spiking and perisynaptic activity on BOLD functional MRI. Proc Natl Acad Sci USA 105: 6759-6764.

Robbins TW, Arnsten AF (2009). The neuropsychopharmacology of fronto-executive function: monoaminergic modulation. Annu Rev Neurosci 32: 267-287.

Rubia K, Halari R, Christakou A, Taylor E (2009a). Impulsiveness as a timing disturbance: neurocognitive abnormalities in attention-deficit hyperactivity disorder during temporal processes and normalization with methylphenidate. Philos Trans $R$ Soc Lond B Biol Sci 364: 1919-1931.

Rubia K, Halari R, Cubillo A, Mohammad AM, Brammer M, Taylor E (2009b). Methylphenidate normalises activation and functional connectivity deficits in attention and motivation networks in medication-naive children with ADHD during a rewarded continuous performance task. Neuropharmacology 57: 640-652.

Rubia K, Halari R, Cubillo A, Smith AB, Mohammad AM, Brammer $\mathrm{M}$ et al (2011a). Methylphenidate normalizes frontostriatal underactivation during interference inhibition in medication-naive boys with attention-deficit hyperactivity disorder. Neuropsychopharmacol 36: 1575-1586.

Rubia K, Halari R, Mohammad AM, Taylor E, Brammer M (2011b). Methylphenidate normalizes frontocingulate underactivation during error processing in attention-deficit/hyperactivity disorder. Biol Psychiatry 70: 255-262.

Schweitzer JB, Lee DO, Hanford RB, Tagamets MA, Hoffman JM, Grafton ST et al (2003). A positron emission tomography study of methylphenidate in adults with ADHD: alterations in resting blood flow and predicting treatment response. Neuropsychopharmacol 28: 967-973.
Sheridan MA, Hinshaw S, D’Esposito M (2010). Stimulant medication and prefrontal functional connectivity during working memory in ADHD: a preliminary report. J Atten Disord 14: 69-78.

Song XW, Dong ZY, Long XY, Li SF, Zuo XN, Zhu CZ et al (2011). REST: a toolkit for resting-state functional magnetic resonance imaging data processing. PLoS One 6: 25031.

Tian L, Jiang T, Liang M, Zang Y, He Y, Sui M et al (2008). Enhanced resting-state brain activities in ADHD patients: a fMRI study. Brain Dev 30: 342-348.

Tian L, Ren J, Zang Y (2012). Regional homogeneity of resting state fMRI signals predicts Stop signal task performance. Neuroimage 60: $539-544$

Vaidya CJ, Austin G, Kirkorian G, Ridlehuber HW, Desmond JE, Glover GH et al (1998). Selective effects of methylphenidate in attention deficit hyperactivity disorder: a functional magnetic resonance study. Proc Natl Acad Sci USA 95: 14494-14499.

Volkow ND, Wang GJ, Fowler JS, Thanos PP, Logan J, Gatley SJ et al (2002). Brain DA D2 receptors predict reinforcing effects of stimulants in humans: replication study. Synapse 46: 79-82.

Wang L, Zhu C, He Y, Zang Y, Cao Q, Zhang H et al (2009). Altered small-world brain functional networks in children with attention-deficit/hyperactivity disorder. Hum Brain Mapp 30: 638-649.

Zang Y, Jiang T, Lu Y, He Y, Tian L (2004). Regional homogeneity approach to fMRI data analysis. Neuroimage 22: 394-400.

Zang YF, He Y, Zhu CZ, Cao QJ, Sui MQ, Liang M et al (2007). Altered baseline brain activity in children with ADHD revealed by resting-state functional MRI. Brain Dev 29: 83-91.

Zhong Y, Lu G, Zhang Z, Jiao Q, Li K, Liu Y (2011). Altered regional synchronization in epileptic patients with generalized tonic-clonic seizures. Epilepsy Res 97: 83-91.

Supplementary Information accompanies the paper on the Neuropsychopharmacology website (http://www.nature.com/npp) 